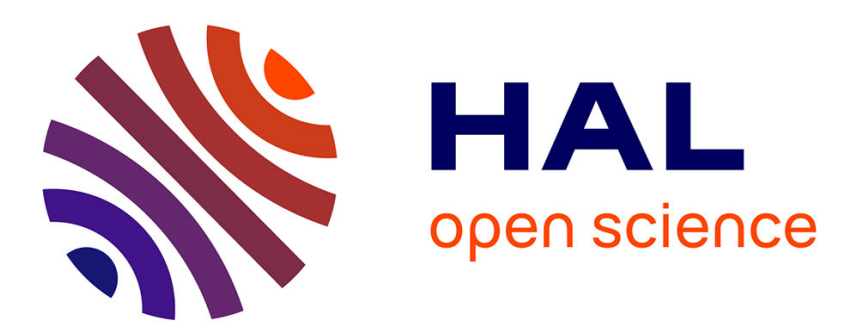

\title{
A Novel Intermolecular Potential to Describe the Interaction Between the Azide Anion and Carbon Nanotubes
}

Stefano Battaglia, Stefano Evangelisti, Thierry Leininger, Fernando Pirani, Noelia Faginas Lago

\section{To cite this version:}

Stefano Battaglia, Stefano Evangelisti, Thierry Leininger, Fernando Pirani, Noelia Faginas Lago. A Novel Intermolecular Potential to Describe the Interaction Between the Azide Anion and Carbon Nanotubes. Diamond and Related Materials, 2020, 101, pp.107533. 10.1016/j.diamond.2019.107533 . hal-02318944

\section{HAL Id: hal-02318944 https://hal.science/hal-02318944}

Submitted on 22 Oct 2019

HAL is a multi-disciplinary open access archive for the deposit and dissemination of scientific research documents, whether they are published or not. The documents may come from teaching and research institutions in France or abroad, or from public or private research centers.
L'archive ouverte pluridisciplinaire HAL, est destinée au dépôt et à la diffusion de documents scientifiques de niveau recherche, publiés ou non, émanant des établissements d'enseignement et de recherche français ou étrangers, des laboratoires publics ou privés. 


\title{
A Novel Intermolecular Potential to Describe the Interaction Between the Azide Anion and Carbon Nanotubes
}

\author{
Stefano Battaglia ${ }^{\mathrm{a}, \mathrm{b}, 1}$, Stefano Evangelisti ${ }^{\mathrm{a}, 2}$, Thierry Leininger ${ }^{\mathrm{a}, 3}$, Fernando \\ Pirani $^{\text {b,c }}$, Noelia Faginas-Lago ${ }^{\text {b,4,* }}$ \\ ${ }^{a}$ Laboratoire de Chimie et Physique Quantiques, IRSAMC, Université de Toulouse et \\ CNRS, 118 Route de Narbonne, F-31062 Toulouse Cedex, France \\ ${ }^{b}$ Dipartimento di Chimica, Biologia e Biotecnologie, Università degli Studi di Perugia, \\ Via Elce di Sotto 8, I-06123 Perugia, Italy \\ ${ }^{c}$ Istituto di Scienze e Tecnologie Molecolari CNR (CNR-ISTM), Perugia - Italy
}

\section{Abstract}

In this contribution we propose a novel and accurate intermolecular potential that can be used for the simulation of the azide anion confined inside carbon nanotubes of arbitrary size. The peculiarity of our approach is to include an explicit term, modeling the induction attractive contributions from the negatively charged azide ion, that can be generalized to other ions confined in carbon nanotubes of different size and length. Through a series of accurate DLPNO-CCSD $(\mathrm{T})$ calculations, we show that this potential reproduces the $a b$ initio interaction energy to within a few $\mathrm{kcal} / \mathrm{mol}$. The potential is implemented in a molecular dynamics program, with which we carried out illustrative simulations to demonstrate the effectiveness of our approach. At last, the guidelines provided by this investigation can be applied to build up force fields for many neutral/ionic molecular species confined within carbon

\footnotetext{
*Corresponding author

Email address: noelia.faginaslago@unipg.it (Noelia Faginas-Lago)

${ }^{1}$ ORCID: 0000-0002-5082-2681

${ }^{2}$ ORCID: 0000-0001-8782-443X

${ }^{3}$ ORCID: 0000-0002-7373-0966

${ }^{4}$ ORCID: 0000-0002-4056-3364
} 
nanotubes; a crucial requirement to carry out molecular dynamics simulations under a variety of conditions.

Keywords: Azide Anion, Carbon Nanotubes, Induction, Intermolecular Potential

\section{Introduction}

The hollow structure of carbon nanotubes (CNTs) and the low reactivity of the internal wall provide an ideal environment to confine a wide variety of systems in their cavity, making them suitable for different types of applications. Since 1993, when Ajayan and Iijima [1] experimentally demonstrated the possibility to fill CNTs by capillary suction proving feasible the early theoretical predictions, a lot of work has been carried out in this domain such that a large variety of confined and composite systems have now been investigated; e.g. fullerenes and metallofullerenes[2, 3], a long list of different metals[4-10], energetic molecules[11-14], and many more. The limited space inside the cavity of CNTs can also induce new phases of materials, with the most notable example being certainly that of water. Novel "ice" phases arising from encapsulated water were first predicted from theoretical calculations[15] and then observed experimentally over the years[16-18].

In the search for alternative and environmentally friendly energy sources, a promising class of systems is that of all-nitrogen molecules[19, 20]. Accounting for as much as $78 \%$ of the total, nitrogen is the most abundant species in Earth's atmosphere and is therefore widely and easily accessible. It is virtually only present as di-molecular $\mathrm{N}_{2}$, which is by far the most stable form and it is not harmful for the environment. The energy difference between single, double and triple covalent bond is such that polynitrogen molecules store an enormous amount of energy and for this reason pure nitrogen clusters are considered as high energy-density materials (HEDM)[19, 20].

The first and most famous polynitrogen molecule is the azide anion, $\mathrm{N}_{3}^{-}$, known since the end of the nineteenth century[21]. It took more than a 
100 years to enlarge this family, with the successful synthesis of the $\mathrm{N}_{5}^{+}$ cation[22] in 1999, renewing the interest in this class of compounds as people envisioned the possibility of a pure nitrogen crystal composed by $\mathrm{N}_{3}^{-}$and $\mathrm{N}_{5}^{+}$ ion pairs[23-25]. However, experimental attempts to stabilize the two ions resulted unsuccessful[26], highlighting their intrinsic instability with respect to decomposition into $\mathrm{N}_{2}$ as the major obstacle towards the realization of pure nitrogen allotropes.

More recently, molecular confinement was explored as a possible strategy to overcome this problem. By encapsulating a polymeric nitrogen chain inside CNTs, it was predicted by molecular dynamics simulations that the nitrogen species remains stable up to high temperatures, preserving, in theory, the structure of these molecules and providing at the same time a way to store them $[12,13,27]$. Additional theoretical investigations based on the same idea predicted the same nitrogen chain to be stable in silicon carbide and boron nitride nanotubes[28-30]. With a similar approach, a comprehensive density functional theory (DFT) study on the encapsulation of nitrogen clusters of different sizes in a $\mathrm{C}_{60}$ fullerene was carried out too, predicting stable structures for molecules of up to 13 atoms before the latter started to be chemically bound to the confining cage[31].

From an experimental perspective, an $\mathrm{N}_{8}^{-}$anionic species adsorbed on the inner side of a multi-wall carbon nanotube was observed at ambient conditions[32]; a result that was recently corroborated by the successful synthesis on a carbon nanotube substrate of the cubic gauche phase $(\mathrm{cg}-\mathrm{N})$ near ambient conditions[33]. In the latter work, besides the presence of signature peaks in Raman and infrared spectra corresponding to the cg-N phase, high resolution transmission electron microscopy images clearly show the encapsulated nitrogen clusters.

Albeit the relatively large number of works on nitrogen chains, not so many other species were considered in a confined environment. In particular, despite the extensive literature investigating the spectroscopic features 
of the azide ion in crystal or gas phase[34-42] as well as solvated[43-55], $\mathrm{N}_{3}^{-}$ was never studied in a confined environment. Considering that $\mathrm{N}_{3}^{-}$often represents a precursor in the synthesis of energetic molecules, e.g. the aforementioned cg-N phase synthesis starts from sodium azide[33], or it appears as an intermediate structure during the dissociation reaction of larger nitrogen clusters, e.g. the barrierless dissociation of the $\mathrm{N}_{5}^{-}$ion $[26,56]$, it is certainly of great interest to study its behavior in a constrained environment such as that provided by carbon nanotubes, and in particular to understand the type of interactions between the confining and the confined fragments.

In this work, the confinement of the azide anion inside carbon nanotubes of different lengths and diameters are presented and a newly developed intermolecular potential is reported. A first part of this manuscript is devoted to an $a b$ initio study of this system, providing important insight into the adsorption of the polynitrogen molecule in the cavity, followed by a systematic investigation where the finite size effects are studied by high-level coupledcluster calculations, providing accurate, reference energies.

Building on the knowledge acquired from the static calculations and comparing to the reference $a b$ initio data, a novel intermolecular potential modeling the non-covalent interaction between $\mathrm{N}_{3}^{-}$and carbon nanotubes is then derived and presented. The potential is implemented in a local version of the molecular dynamics (MD) program DL POLY 4.08[57, 58] and the working equations of the potential are reported here. The implementation of this potential allows to accurately study the dynamics of the confined ion in carbon nanotubes of any size and type, and in principle solvated in any non-ionic liquid. The implementation of the potential is tested by performing simulation of the confined system which reproduce well the ab initio results. Moreover, we should emphasize that the methodology proposed here to characterize static and dynamical properties of the prototype $\mathrm{N}_{3}^{-}$molecular ion confined in CNTs can be also generalized to build up the force fields (FF) for many other neutral/ionic molecular species encapsulated in CNTs. The availability 
of $\mathrm{FF}$ given in analytical form is a crucial condition to carry out molecular dynamics simulations under a variety of conditions and it is therefore of general interest for the characterization of the behavior of prototype polyatomic molecular ions confined in a nanotube.

The paper is structured as follows. In section 2 we present the ab initio results along with the computational details of the performed calculations. In section 3 we focus on the derivation of the intermolecular potential and the parameters entering its formulation. In section 4 we present the results of MD simulations carried out to test the potential and finally in section 5 we draw the conclusions of this study.

\section{Ab initio Calculations}

In order to propose the new intermolecular potential, we first carried out a series of $a b$ initio calculations. In this part, carbon nanotubes were treated as finite-size systems, with the addition of hydrogen atoms at both ends in order to fill the valence shell. Only the armchair $\operatorname{CNT}(5,5)$ was considered, as this was previously reported to be the CNT showing strongest interaction with the azide anion[14]. Clearly, the interaction between the CNT and the azide strongly depends on the length of the former, a detail that is often not discussed in similar studies. Thus, in order to obtain converged values with respect to the length of the nanotube, CNTs with different number of units were used. We shall use for the rest of this article the notation $\Lambda$-CNT $(5,5)$ to label a nanotube composed by $\Lambda$ units, where a single unit corresponds to a $[10]$ cyclophenacene.

\subsection{Geometry}

The geometries of the nanotubes and the azide anion were optimized using restricted Kohn-Sham DFT, employing the B97D3 exchange-correlation functional with Becke-Johnson damping[59, 60]. For both fragments, Dun-

ning's triple- $\zeta$ cc-pVTZ basis set[61] was used, with the addition of diffuse 
functions (aug-) [62] in the case of the $\mathrm{N}_{3}^{-}$fragment. The nanotubes geometries retained the high $D_{5 d}$ symmetry, with both parallel and perpendicular (to the principal axis) $\mathrm{C}-\mathrm{C}$ bonds showing the typical alternation pattern of these systems when treated with open boundary conditions[63, 64]. As the length of the nanotube increases, the average $\mathrm{C}-\mathrm{C}$ bond length also increases, from $1.4237 \AA$ to $1.4268 \AA$, which is in agreement with the fact that the bond gets slightly stretched due to the curvature. The relaxed $\mathrm{N}_{3}^{-}$structure has linear $D_{\infty h}$ symmetry, with an $\mathrm{N}-\mathrm{N}$ bond length of $1.1874 \AA$ in excellent agreement with the experimental gas phase value of $1.1884 \AA[37]$.

All these optimizations were performed using the Gaussian 09 software package, revision d01[65], using the default convergence thresholds, the ultrafine grid for the integral quadrature and spherical $d$ and $f$ basis functions.

\subsection{Interaction Energy}

The ab initio interaction energies were calculated according to the following formula

$$
E_{\text {int }}=E_{\text {complex }}-\left(E_{\text {cnt }}+E_{\text {azide }}\right)
$$

where the fragment energies $E_{c n t}$ and $E_{\text {azide }}$ are obtained in the basis set of the complex system accounting for the basis set superposition error following the Boys and Bernardi scheme[66]. Relaxation effects are not taken into account, as these were recently shown to be in the order of $1-2 \mathrm{kcal} / \mathrm{mol}$ only[14]. To locate the minimum of the potential energy surface (PES), a series of constrained optimizations involving nanotubes of lengths between 3 and 9 units was performed at the same level of theory as before, despite employing the smaller cc-pVDZ basis set on the nanotube and the resolution of the identity approximation using the auxiliary basis set def2/J by Weigend [67]. Differently to the previous case, these calculations were per-

formed using the ORCA 4 program package[68] (version 4.0.0). In these calculations, the optimal geometry of the two fragments (i.e. the one obtained by B97D3/(aug-)cc-pVTZ) was kept frozen and only their relative 
distance and orientation were relaxed, thus locating the optimal adsorption distance. One can imagine that, given the highly periodic structure of the carbon nanotubes, the number of minima in the PES is extremely large, however many of those are expected to be quite close in energy. Remarkably, it turns out that in the particular case of an azide anion confined inside a $\mathrm{CNT}(5,5)$, the geometry with the $\mathrm{N}_{3}^{-}$molecule placed exactly in the center of the nanotube and aligned to its principal axis results to be a local minimum. The lowest minimum found with this procedure is nevertheless very similar, with the azide anion still nearly perfectly aligned to the principal axis, but displaced by approximately $0.5 \AA$ towards one of the two openings. The energy difference between these two minima is negligible (less than 0.6 $\mathrm{kcal} / \mathrm{mol}$ ) and thus, for the sake of simplicity, the geometry with the $\mathrm{N}_{3}^{-}$ion placed in the center of the CNT was used for the calculation of $E_{\text {int }}$ at higher levels of theory and for nanotubes longer than 9 units.

As already mentioned before, it is important to study the dependence of the interaction energy with respect to the length of the nanotube. In particular, given that the confined species is a negatively charged ion, we should expect a slower convergence of this quantity compared to uncharged systems due to the more diffuse electron density. To obtain highly accurate interaction energies for systems as large as the carbon nanotubes studied here, approximations to canonical post-Hartree-Fock methods were employed. In particular, the energy were obtained using the density-fitted spin-componentscaled MP2 method (RI-SCS-MP2)[69] and the local coupled cluster method DLPNO-CCSD(T) as implemented in the ORCA 4 program package[70, 71]. The cc-pVTZ basis set was used where computationally feasible, otherwise the cc-pVDZ one. Diffuse functions (aug-) were added on the nitrogen atoms in both cases. In order to assess the basis set incompleteness error, for the nanotube with $\Lambda=3$, we have carried out an RI-SCS-MP2 calculation using the larger (aug-)cc-pVQZ basis set and extrapolated the results to the complete basis set limit (CBS) using the triple- and quadrupole- $\zeta$ bases energies. 
In particular, for each fragment, we have extrapolated the RI-SCS-MP2 correlation energy (i.e. not the total RI-SCS-MP2 energy), labeled and then computed the interaction energy using the CBS values. In order to do so, we have used the two-point extrapolation scheme by Halkier et al. [72], with the formula

$$
E_{\text {corr }}(\infty)=\frac{E(X) X^{3}-E(Y) Y^{3}}{X^{3}-Y^{3}}
$$

where $X$ and $Y$ are the cardinal numbers of the basis set $(X=3$ and $Y=$ 4 for triple- and quadrupole- $\zeta$, respectively) and $E(X)$ and $E(Y)$ are the corresponding energies. The interaction energies obtained for the three basis sets (we show also the values obtained with the double- $\zeta$ basis) and the CBS limit are reported in Table 1. As we can see, going from triple- to quadrupole$\zeta$, the interaction energy changes by less than $1 \mathrm{kcal} / \mathrm{mol}$, whereas going to the CBS limit, the difference with the triple- $\zeta$ is only about $1 \mathrm{kcal} / \mathrm{mol}$. In contrast, we clearly see that the double- $\zeta$ results underestimate substantially the interaction strength. According to this analysis we can conclude that the errors introduced by the finite basis will be very likely less than those introduced by the classical approximation of the force field.

The results obtained with the different methods, using the optimal geometries of the fragments and with the azide anion placed exactly in the center of the CNT, are shown as a function of the nanotube length in Figure 1. For all CNTs long enough to confine the azide anion, the reported energy is negative, meaning a favorable interaction between the nanotube and the guest species (note that the case $\Lambda=1$ is excluded from Figure 1 because given that the CNT is extremely short, the azide anion is in practice not confined anymore and the resulting interaction energy is positive, corresponding to the azide trying to "escape" the nanobelt). From the plot, it is immediately clear how the interaction energy has a slow convergence with respect to the number of units $\Lambda$, a behavior that we shall investigate further.

Coupled-cluster-level energies were obtained in conjunction with a double- $\zeta$ basis set only, whereas a few points at triple- $\zeta$ quality are shown for RI-SCS- 
MP2. By assuming that the correlation energy difference between the two methods is constant with respect to the basis set used, it is possible to extrapolate DLPNO-CCSD $(\mathrm{T})$ interaction energies to the larger cc-pVTZ basis set according to the formula

$$
E_{C C}(p V T Z) \approx E_{M P 2}(p V T Z)+\left(E_{C C}(p V D Z)-E_{M P 2}(p V D Z)\right)
$$

where $E_{C C}$ and $E_{M P 2}$ refer to the DLPNO-CCSD(T) and RI-SCS-MP2 energies, respectively. This approach has allowed us to obtain approximate DLPNO-CCSD(T)/cc-pVTZ values for nanotubes of length $\Lambda=3,5$ and 7 units. Nevertheless, as it can be seen from the RI-SCS-MP2/cc-pVDZ points, $E_{\text {int }}$ still significantly decreases with the addition of extra units beyond the seventh. Therefore, following the approach taken by Grimme et al. [73], we have fitted the three approximate DLPNO-CCSD $(\mathrm{T}) / \mathrm{cc}-\mathrm{pVTZ}$ energies to the following function

$$
E_{\text {int }}(\Lambda)=\frac{a \Lambda}{b+\Lambda}
$$

where $a$ and $b$ are two fitting parameters to be determined and $\Lambda$ is the number of nanotube units. Equation (4) is the simplest formula which satisfies the condition $E_{\text {int }}(0)=0$ and provides an estimate for the infinitely long nanotube through the parameter $a$. The dashed lines in Figure 1 show the non-linear least square fits to the RI-SCS-MP2/cc-pVDZ and the approximate DLPNO-CCSD $(\mathrm{T}) / \mathrm{cc}-\mathrm{pVTZ}$ interaction energies, respectively. The optimized parameters are reported in Table 2 , where it can be seen how the values $a=-65.93 \mathrm{kcal} / \mathrm{mol}$ and $a=-64.75 \mathrm{kcal} / \mathrm{mol}$, i.e. the asymptotic value of the interaction energy, are remarkably close to each other despite being obtained with different quantum chemical methods.

\section{Intermolecular Potential}

Building on a previous in-depth analysis on the interaction nature between the azide anion and carbon nanotubes[14], three main types of inter- 
action (in classical terms) were identified for the system under study: van der Waals (vdW) interactions (these are always present), induction effects due to the charged $\mathrm{N}_{3}^{-}$fragment and electrostatic effects between the polarized nanotube and the anion. We shall see how these different interactions can be accurately modeled and added together to form a complete description of all non-covalent interactions present in the system.

\subsection{Model Potential Definition}

Van der Waals interactions are modeled according to the Improved LennardJones (ILJ) potential, a modified form of the canonical Lennard-Jones potential proposed a few years ago by Pirani et al. [74]. The general formula reads

$$
V_{i l j}\left(r_{i j}\right)=\epsilon\left[\frac{m}{n\left(r_{i j}\right)-m}\left(\frac{r_{m}}{r_{i j}}\right)^{n\left(r_{i j}\right)}-\frac{n\left(r_{i j}\right)}{n\left(r_{i j}\right)-m}\left(\frac{r_{m}}{r_{i j}}\right)^{m}\right]
$$

where

$$
n\left(r_{i j}\right)=\beta+4.0\left(\frac{r_{i j}}{r_{m}}\right)^{2}
$$

The indices $i$ and $j$ refer to atoms of the system which do not belong to the same molecule. For each unique pair of interacting atom types, there are three parameters to be set. The value $r_{m}$ corresponds to the position of the minimum of the potential energy surface for the interaction of the two atoms labeled by $i$ and $j$. The depth of that minmum is given by the value of $\epsilon$ and the last parameter is $\beta$, appearing in Equation (6), which is related to the hardness of the system and usually varies between 7 and 9[74]. The value of $m$ entering Equation (5) as an exponent and a prefactor is set according to the type and charge of the interacting centers: for atom-atom interactions $m$ is equal 6 , for ion-atom interactions is equal 4 and for ion-ion is set to 1 . This is therefore not a freely varying parameter, but a value which is set a priori.

The ILJ potential has proven very effective in a number of applications, 
in particular dealing with polar molecules such as water[75], water-carbon systems[76], ion- $\pi$ interactions[77, 78], and in general gases interacting with carbon nanostructures such as graphene and carbon nanotubes[79-84].

Albeit the ability of the ILJ potential to accurately reproduce the potential energy surface of interacting ion-neutral species, it is most often the case that the ions in question are individual atoms; this fact allows for a pairwise treatment of the interaction as it is done, e.g., in the ILJ potential. However, the presence of arbitrary, non-spherically-symmetric molecular ions substantially complicate this picture. The latter are responsible for strong induction effects acting on the interacting species, which are non-additive in nature[85] and increase the complexity of the model potential.

The induction effects generated by the presence of $\mathrm{N}_{3}^{-}$can be modeled according to a relatively simple formula which arises directly from the general formula of an induced dipole, i.e.

$$
\mu^{*}=\alpha \mathcal{E}
$$

where $\mu^{*}$ is the induced dipole, $\alpha$ is the polarizability and $\mathcal{E}$ is the external electric field. In the system we are considering, the external electric field is the one generated by the partial atomic charges of the azide, whereas the polarizability is that of the carbon nanotube. For the specific case of the electric field generated by three point charges, the induction potential, obtained by integration of Equation (7) is given by

$$
V_{i n d}\left(r_{i 1}, r_{i 2}, r_{i 3}\right)=-\frac{1}{2} \alpha_{i}\left[\frac{q_{1}}{r_{i 1}^{2}}+\frac{q_{2}}{r_{i 2}^{2}}+\frac{q_{3}}{r_{i 3}^{2}}\right]^{2}
$$

where $r_{i 1}, r_{i 2}$ and $r_{i 3}$ are the distances between atom $i$ of the nanotube and the three nitrogen atoms of the azide anion, $q_{1}, q_{2}$ and $q_{3}$ are their partial atomic charges and $\alpha_{i}$ is the polarizability per volume of atom $i$. Note that the choice to model the electric field with three partial charges, one for each 
nitrogen center, is arbitrary, nevertheless the most natural. The fact that the electric field is generated by more than one charge is the mathematical reason why the induction potential is non-additive.

The last type of interaction occuring for this system is the electrostatic one. By using finite, hydrogen-saturated nanotubes, the CNT wall gets polarized and thus, within a classical description of the interaction, electrostatic effects between this charge accumulation at the extremities and the azide anion are non-negligible. This type of interaction is quite standard and is usually modeled by the Coulomb potential, given by

$$
V_{e l s}\left(r_{i j}\right)=\frac{q_{i} q_{j}}{r_{i j}}
$$

where $q_{i}$ and $q_{j}$ are the charges of the interacting atomic centers and $r_{i j}$ is the distance between them. Note that in the absence of hydrogens, the nanotube remains in principle completely unpolarized, hence no partial charges are assigned to the carbon atoms. This makes the contribution of $V_{\text {els }}$ exactly zero.

The total intermolecular potential describing the interaction between the azide anion and an arbitrary carbon nanotube is given by the combination of the three components just introduced, summing over all atoms of the interacting fragments, i.e.

$$
V_{\text {tot }}=\sum_{i=1}^{N_{c n t}} V_{i n d}^{(i)}\left(r_{i 1}, r_{i 2}, r_{i 3}\right)+\sum_{i=1}^{N_{a z i d e}} \sum_{j=1}^{N_{c n t}} V_{i l j}^{(i j)}\left(r_{i j}\right)+V_{e l s}\left(r_{i j}\right)
$$

where $N_{\text {cnt }}, N_{\text {azide }}$ correspond to the total number of atoms in the carbon nanotube and the azide anion, respectively. The superscript $(i j)$ in the ILJ potential term $V_{i l j}^{(i j)}$ labels the type of interacting atoms, since for every unique pair there is a different set of parameters. 


\subsection{Model Potential Parameters}

With this choice of potential, we are now set to test its accuracy by comparing the interaction energies obtained with the DLPNO-CCSD $(\mathrm{T})$ method with those obtained from Equation (10). In this study, we used a combination of experimental and $a b$ initio data, together with correlation formulas derived from extensive empirical studies[86-88] to obtain the parameters used in the potential function. The most important parameters are the atomic polarizabilities, which are used to determine the $\epsilon$ and $r_{m}$ values for $V_{i l j}$, as well as appearing directly in the induction potential. The nitrogen atomic polarizability is obtained from the experimental total mean polarizability of the azide anion[89], $\bar{\alpha}=4.65 \AA^{3}$, which was distributed on the three atoms according to their partial charges, resulting in a value of $1.9 \AA^{3}$ for the external atoms (charges $q_{1}=q_{3}=-0.56$ atomic units $(\mathrm{au})$ ) and a value of $0.85 \AA^{3}$ for the internal one (charge $q_{2}=+0.12 \mathrm{au}$ ). The polarizability of the hydrogen atoms was taken from Ref. 90 and set equal to $0.380 \AA^{3}$, whereas that of carbon atoms from Ref. 91, where we note that the actual value depends on the specific curvature (diameter) of the nanotube. The polarizability for carbon atoms, along with the calculated ILJ parameters according to Ref. 86 are reported in Table 3. Similarly, the parameters for hydrogen and the hydrogen-nitrogen interaction are listed in Table 4. Atomic partial charges for the carbon nanotube were obtained through a natural population analysis (NPA)[92] of the electron density calculated at B97D3/cc-pVTZ level of theory. Instead, those for the nitrogen atoms in the the electrostatic and induction potentials were set equal to $q_{1}=q_{3}=-0.56 \mathrm{au}$ (external atoms) and $q_{2}=+0.12$ au (internal atom) according to the $a b$ initio values reported by Le Borgne et al. [89]. As a matter of comparison, an NPA of $\mathrm{N}_{3}^{-}$obtained with B97D3/aug-cc-pVTZ (both geometry and electron density) results in partial charges $q_{1}=q_{3}=-0.55$ au and $q_{2}=+0.10 \mathrm{au}$. 


\subsection{Potential Energy Minimum and Profile}

To assess the accuracy of the potential energy function we compare the interaction energy obtained using the ab initio methodology and the one obtained with the model potential for all nanotubes considered so far. In Figure 2 the $a b$ initio and the classical potential energies are reported for $\beta=8$ as a function of the nanotube length $\Lambda$. As can be seen, for the shortest nanotube, the classical potential largely underestimates the interaction strength, however, starting from 7 units, the agreement is excellent. The fact that different types of interactions are explicitly considered naturally allows for an energy decomposition analysis. The plot depicted in Figure 3 shows the same two curves as in Figure 2, however, the relative contributions of the three components $V_{i l j}, V_{\text {ind }}$ and $V_{\text {els }}$ are also reported. It is interesting to see the different ranges of the types of interaction. The vdW contribution described by the ILJ potential account for only a fraction of the total interaction energy. Moreover, the convergence with respect to the length of the nanotube is essentially immediate. Despite the fact that vdW interactions are usually called long-range in the quantum chemistry context, because exchange interactions usually decay exponentially as function of the distance, in this framework they actually should be considered as short-range due to their $r^{-6}$ dependence. The induction potential appears to converge also quite quickly with respect to the nanotube length. In this case however, the dependence is given by the inverse fourth power of the distance. Here, the magnitude of this contribution is very surprising; the explicit modeling of this interaction seems therefore crucial in order to provide a good description of the potential well. At last, the longest-range interaction is given by the Coulomb potential, whose strength is inversely proportional to the distance between the atomic centers. From this component it is clear how the shape of the total classical potential represented in Figure 2 is dominated by this term. Since between 5 and 7 units, the improved Lennard-Jones and the induction potentials are essentially constant, the convergence behavior of the 
three terms summed is solely determined by the electrostatics.

Thanks to the analytical form of the intermolecular potential, the interaction energy profile of the azide ion moving inside and outside nanotubes of arbitrary size can be generated. As an example, we considered in the following three 7 -unit long nanotubes, namely $(5,5),(7,7)$ and $(9,9)$ CNTs. The parameters used for each nanotube are listed in Table 3 and the partial charges assigned to the carbon an hydrogen were obtained again by a natural population analysis[92] using the electron density from a B97D3/cc-pVTZ calculation. We investigated the profiles of the azide anion moving in three different ways. In the first case, $\mathrm{N}_{3}^{-}$was set parallel to the nanotube axis and moved along the $x$ direction, in the second case it was passed through the nanotube along the $z$ axis at the optimal adsorption distance found in the first profile and in the last case case it was fixed at the center and rotated. A scheme representing the 3 different situations is shown in Figure 4 . The first potential energy surface that we investigated is that generated along the $x$ axis, such that the optimal adsorption distances can be identified. In Figure 5 the results for the three nanotubes are shown stacked on top of each other, starting with the narrowest from the top. The most favorable interaction is always observed inside the CNT, even though a minimum is also found on the external side of the wall in all cases. A double well appears inside the cavity for the two largest systems, whereas the optimal adsorption distance for the $\operatorname{CNT}(5,5)$ is exactly at the center as was previously found by the $a b$ initio calculations. The different components of the potential behave very similarly across the three systems. Remarkably, by going from the smallest to the largest systems, the overall shape of the total interaction resembles more that of the van der Waals potential, in particular inside the cavity.

In the second case, the profile along the nanotube axis was investigated. For the $\mathrm{CNT}(5,5)$, the azide position coincided with the nanotube axis, for the $(7,7) \mathrm{CNT}$ it was placed at a distance of $3.07 \AA$ from the wall, and for the $(9,9)$ nanotube the distance was $3.20 \AA$. Note that this distance corresponds 
to the minimum of the potential well obtained in the first case; i.e. the optimal adsorption distance of the azide anion confined in the carbon nanotubes considered here. The corresponding profiles are shown in Figure 6, where as before the plots are arranged on top of each other, starting from the top with the $\mathrm{CNT}(5,5)$. In all cases, if initially placed outside, the azide anion is attracted towards the nanotube and depending on its relative position with respect to the CNT axis a local minimum is present. An interesting result is the flipping of electrostatic interaction, which is attractive when the azide is outside the CNT since the external hydrogen atoms are positively charged, and repulsive inside since there is excess negative charge on the carbons.

The last case analyzed is the rotation inside the nanotube reported in Figure 7. This last series of profiles shows very clearly the effects of spatial confinement provided by the enclosing CNT. In a $\mathrm{CNT}(5,5)$ the ion can barely rotate and tremendous repulsive walls build around the minimum of the PES. On the other hand, already with the slightly larger $\operatorname{CNT}(7,7)$ the (repulsive) effects are only mildly perceived by the ion. For the largest nanotube, the potential is basically flat, suggesting that a single $\mathrm{N}_{3}^{-}$ion is likely to freely rotate even when confined.

\section{Molecular Dynamics Simulations}

The intermolecular potential presented in the previous section was implemented in a locally modified version of the DL POLY 4.08 program[57, 58]. In particular, analytical energy gradients were derived for both the ILJ and the induction potentials, whereas the Coulomb potential is already available as part of the original software. It should be noted that the induction potential, being non-additive in nature, is forcedly hard-coded for the specific case of an electric field generated by three point charges. Nevertheless, there is no particular assumption towards the azide anion: any fragment modeled by three point charges can make use of this potential.

In order to validate the implementation, a series of MD simulations was 
carried out on the same systems for which the energy profiles were presented, i.e. the azide anion confined in a $\operatorname{CNT}(5,5)$, a $\operatorname{CNT}(7,7)$ and a $\operatorname{CNT}(9,9)$. In this case however, the carbon nanotubes were considered with PBC and hence without addition of hydrogen atoms at the two ends. This removes the need of electrostatic interactions, since the nanotube is not polarized.

The simulations were carried in the canonical ensemble (NVT), using the Nosé-Hoover thermostat with a relaxation constant of 0.5 ps at a temperature of $300 \mathrm{~K}$. Orthorombic periodic boundary conditions were applied, with a simulation box size of $40 \times 40 \times 32.0036 \AA^{3}$, where the CNT principal axis was aligned to the shortest edge of the box and centered with respect to the other two dimensions. We carried out an equilibration phase of 2 ns and a production phase of $3 \mathrm{~ns}$, using a timestep of 1 fs. Statistical sampling was recorded every 10 ps. To minimize the number of parameters in these simulations, the two fragments were considered frozen for the entire simulation such that no intramolecular potential was necessary. However, the introduction of flexibility in the nanotube and the azide anion are easily possible. For each nanotube we repeated the simulation 10 times, each time starting from a new, randomly generated position of the azide ion inside the nanotube.

In Figure 8 we report the average adsorption energy as a function of time. Although the fluctuations for individual runs are quite severe due to the rigid-body approximation and to the use of a single azide ion, the adsorption energy averaged over the 10 runs started from different configurations is significantly smoother. The mean energy values (i.e. the temporal average of the adsorption energy) is $-62.98,-42.32$ and -34.77 (in $\mathrm{kcal} / \mathrm{mol}$ ) for $\mathrm{CNT}(5,5), \operatorname{CNT}(7,7)$ and $\mathrm{CNT}(9,9)$, respectively, and is in good agreement with respect to the static calculations $(\mathrm{CNT}(5,5))$. In particular, the extrapolated DLPNO-CCSD $(\mathrm{T}) / \mathrm{cc}-\mathrm{pVTZ}$ interaction energy was estimated to be $-65.93 \mathrm{kcal} / \mathrm{mol}$, thus within only a few $\mathrm{kcal} / \mathrm{mol}$ to the mean energy obtained with the dynamics. The advantage of such an intermolecular potential is that the interaction energy for larger systems such as the $\mathrm{CNT}(7,7)$ 
and $\operatorname{CNT}(9,9)$ can be predicted with a high degree of accuracy; otherwise not possible by employing the same combination of ab initio method and basis set. Furthermore, the availability of this potential in the MD software allows the study of dynamical properties as well. As an example, we report in Figure 9 the average adsorption distance between the azide anion and the CNT wall for the three different diameters, obtained by averaging the distance between each nitrogen atom of $\mathrm{N}_{3}^{-}$and the closest carbon atom of the CNT. Again, the results shown in Figure 9 are averages over the 10 runs started from different initial position of the anion. In this case we note how the average distance does not fluctuate much for the $\operatorname{CNT}(5,5)$ system, whose reason can be easily understood with the help of the profile shown Figure 7. The space inside the cavity is so limited that the azide anion can barely get out of the CNT principal axis, resulting in a relatively constant average adsorption distance. For instance, this is not the case for the other two nanotubes, where fluctuations are clearly larger. Again, considering the central and bottom plots of Figure 7 we note that potential energy function is very shallow, allowing the azide to rotate and thus increasing the fluctuations. A surprising result is that the average adsorption distance is smaller for the larger $\operatorname{CNT}(9,9)$ than for the smaller $\operatorname{CNT}(7,7)$, suggesting that the latter nanotube has just the right diameter for the azide to be constantly moving through the center and be adsorbed all around the cavity. On the contrary, for the $\operatorname{CNT}(9,9)$, the ion seems trapped in a minimum on just one side of the internal wall, without being able to escape it. This result can be explained by the barrier existing between the double minimum appearing in Figure 5. For $\operatorname{CNT}(9,9)$ this is about $20 \mathrm{kcal} / \mathrm{mol}$, whereas for $\operatorname{CNT}(7,7)$ it is only in the order of a few $\mathrm{kcal} / \mathrm{mol}$.

\section{Conclusions}

In this contribution, we have presented a new intermolecular potential energy function that describes the interaction between an azide anion and 
carbon nanotubes. This model is based on a series of high-level ab initio calculations performed specifically for this work as well as on a previous study carried out in our group[14]. This particular potential has been implemented in a local version of the molecular dynamics program DL POLY 4.08[57, 58], with which we have demonstrated its performance with a series of illustrative simulations on the confined azide anion.

On one hand, in the construction of this model, we carried out extensive $a b$ initio calculations which complement the work done in Ref. 14 and in particular we have addressed the very important issue of length dependence in the calculation of interaction energies for species confined in carbon nanotubes. When working with finite-size systems, it is crucial to investigate the behavior, in principle, of any molecular property as a function of the length in order to obtain quantitative results. On the other hand, we have found that MD simulations based on the potential proposed here are able to reproduce the interaction energies obtained with the DLPNO-CCSD $(\mathrm{T})$ method to within a few $\mathrm{kcal} / \mathrm{mol}$, which is a small fraction of the total interaction energy. Such a model retains the computational efficiency typical of classical molecular dynamics simulations, providing however a very good accuracy compared to the (much) more expensive $a b$ initio calculations. Several other advantages apply as well. First and foremost the access to the dynamical properties of the system under a variety of conditions: indeed, this potential can be used in conjunction with any type of statistical ensemble. In our particular example, we have computed the mean adsorption distance of the azide anion confined in nanotubes of different diameters at constant volume and temperature, with the surprising result that larger nanotubes may have a more stabilizing effect than smaller ones. Another advantage is the general nature of this potential that can be applied to carbon nanotubes of arbitrary size and dimension, and in the limit even for graphene. In particular, we expect it to be applicable to carbon nanotubes of different chiralities, provided that model parameters are available. The latter can however be obtained through 
experiments, calculations and/or phenomenological methods[76-79]. Open as well as periodic boundary conditions can be handled by this potential, as the electrostatic interaction term can be included or not according to it. As of now, there are two major limitations of the proposed model. First, it has been constructed for single-wall carbon nanotubes only. Its applicability on multi-wall CNTs is therefore at the moment not considered, nor suggested. A second issue is the tradeoff for the accuracy obtained: the explicit modeling of the induction term is of foremost importance in order to obtain such accurate results, however, due to its non-additive nature, this term has been explicitly coded. This means that at present, only induction effects generated by a source of three point charges can be included. Actually, the presence of an explicit term describing induction effects is the key aspect of this work. The latter certainly reduces the transferability of the model proposed, although we should note that by borrowing techniques from the field of machine learning, such as automatic differentiation, it is in principle possible to generalize our approach to handle system with any number of point charges as sources[93].

At last, and perhaps most importantly, this study provides guidelines that can be used to create force fields for other neutral and ionic molecular species confined within carbon nanotubes, that still maintain the analytical form necessary to efficiently carry out molecular dynamics simulations on systems of any dimension.

\section{Acknowledgments}

The results included in this publication have received funding from the European Union's Horizon 2020 research and innovation programme under the Marie Skłodowska-Curie grant agreement $n^{\circ} 642294$. S. E. and T. L. acknowledge the "Programme Investissements d'Avenir" ANR-11-IDEX-000202, reference ANR-10-LABX-0037-NEXT for financial support. N. F.-L. thanks MIUR and the University of Perugia for the financial support of the 
AMIS project through the "Dipartimenti di Eccellenza" programme. N. F.-L. also acknowledges the Fondo Ricerca di Base 2017 (RICBASE2017BALUCANI) del Dipartimento di Chimica, Biologia e Biotecnologie della Università di Perugia for financial support.

\section{References}

[1] P. M. Ajayan, S. Iijima, Capillarity-induced filling of carbon nanotubes, Nature 361 (1993) 333-334. doi:10.1038/361333a0.

[2] B. W. Smith, M. Monthioux, D. E. Luzzi, Encapsulated C60 in carbon nanotubes, Nature 396 (1998) 323-324. doi:10.1038/24521.

[3] J. Lee, H. Kim, S. J. Kahng, G. Kim, Y. W. Son, J. Ihm, H. Kato, Z. W. Wang, T. Okazaki, H. Shinohara, Y. Kuk, Bandgap modulation of carbon nanotubes by encapsulated metallofullerenes, Nature 415 (2002) 1005-1008. doi:10.1038/4151005a.

[4] R. S. Ruoff, D. C. Lorents, B. Chan, R. Malhotra, S. Subramoney, Single Crystal Metals Encapsulated in Carbon Nanoparticles, Science 259 (1993) 346-348. doi:10.1126/science.259.5093.346.

[5] W. Y. Choi, J. W. Kang, H. J. Hwang, Structures of ultrathin copper nanowires encapsulated in carbon nanotubes, Physical Review B 68 (2003) 193405. doi:10.1103/PhysRevB.68.193405.

[6] R. C. Che, L.-M. Peng, X. F. Duan, Q. Chen, X. L. Liang, Microwave Absorption Enhancement and Complex Permittivity and Permeability of Fe Encapsulated within Carbon Nanotubes, Advanced Materials 16 (2004) 401-405. doi:10.1002/adma.200306460.

[7] Z. Liu, Y. Bando, M. Mitome, J. Zhan, Unusual Freezing and Melting of Gallium Encapsulated in Carbon Nanotubes, Physical Review Letters 93 (2004) 095504. URL: 
https://link.aps.org/doi/10.1103/PhysRevLett.93.095504.

doi:10.1103/PhysRevLett.93.095504.

[8] L.-J. Li, A. N. Khlobystov, J. G. Wiltshire, G. A. D. Briggs, R. J. Nicholas, Diameter-selective encapsulation of metallocenes in single-walled carbon nanotubes, Nature Materials 4 (2005) 481-485. doi:10.1038/nmat1396.

[9] B. Xu, B. C. Pan, Study of Gallium Fragments Encapsulated in Single-Walled Carbon Nanotubes, The Journal of Physical Chemistry C 113 (2009) 567-570. URL: $\quad$ http://pubs.acs.org/doi/abs/10.1021/jp807477a. doi:10.1021/jp807477a.

[10] I. Garg, H. Sharma, K. Dharamvir, V. K. Jindal, DFT Study of $\mathrm{Al}_{n}(1-13)$ Clusters Encapsulated Inside Single Walled Carbon Nanotubes, The Journal of Physical Chemistry C 114 (2010) 18762-18772. doi:10.1021/jp1036475.

[11] M. Smeu, F. Zahid, W. Ji, H. Guo, M. Jaidann, H. AbouRachid, Energetic Molecules Encapsulated Inside Carbon Nanotubes and between Graphene Layers: DFT Calculations, Journal of Physical Chemistry C 115 (2011) 1098510989. URL: http://pubs.acs.org/doi/abs/10.1021/jp201756p. doi:10.1021/jp201756p.

[12] H. Abou-Rachid, A. Hu, V. Timoshevskii, Y. Song, L.-S. Lussier, Nanoscale High Energetic Materials: A Polymeric Nitrogen Chain $\mathrm{N}_{8}$ Confined inside a Carbon Nanotube, Physical Review Letters 100 (2008) 196401. doi:10.1103/PhysRevLett.100.196401.

[13] W. Ji, V. Timoshevskii, H. Guo, H. Abou-Rachid, L. Lussier, Thermal stability and formation barrier of a high-energetic material $\mathrm{N}_{8}$ polymer 
nitrogen encapsulated in $(5,5)$ carbon nanotube, Applied Physics Letters 95 (2009) 021904. doi:10.1063/1.3162334.

[14] S. Battaglia, S. Evangelisti, N. Faginas-Lago, T. Leininger, $\mathrm{N}_{3}^{-}$azide anion confined inside finite-size carbon nanotubes, Journal of Molecular Modeling 23 (2017) 294. doi:10.1007/s00894-017-3468-8.

[15] K. Koga, G. T. Gao, H. Tanaka, X. C. Zeng, Formation of ordered ice nanotubes inside carbon nanotubes, Nature 412 (2001) 802-805. doi:10.1038/35090532.

[16] Y. Maniwa, H. Kataura, M. Abe, S. Suzuki, Y. Achiba, H. Kira, K. Matsuda, Phase Transition in Confined Water Inside Carbon Nanotubes, Journal of the Physical Society of Japan 71 (2002) 2863-2866. doi:10.1143/JPSJ.71.2863.

[17] Y. Maniwa, H. Kataura, M. Abe, A. Udaka, S. Suzuki, Y. Achiba, H. Kira, K. Matsuda, H. Kadowaki, Y. Okabe, Ordered water inside carbon nanotubes: formation of pentagonal to octagonal ice-nanotubes, Chemical Physics Letters 401 (2005) 534-538. doi:10.1016/j.cplett.2004.11.112.

[18] K. V. Agrawal, S. Shimizu, L. W. Drahushuk, D. Kilcoyne, M. S. Strano, Observation of extreme phase transition temperatures of water confined inside isolated carbon nanotubes, Nature Nanotechnology 12 (2017) 267-273. doi:10.1038/nnano.2016.254.

[19] P. C. Samartzis, A. M. Wodtke, All-nitrogen chemistry: how far are we from $\mathrm{N}_{60}$ ?, International Reviews in Physical Chemistry 25 (2006) 527-552. doi:10.1080/01442350600879319.

[20] V. E. Zarko, Searching for Ways to Create Energetic Materials Based on Polynitrogen Compounds (Review), Combustion, Explosion and Shock Waves 46 (2010) 121-131. doi:10.1007/s10573-010-0020-x. 
[21] T. Curtius, Ueber Stickstoffwasserstoffsäure (Azoimid) $\mathrm{N}_{3} \mathrm{H}$, Berichte der deutschen chemischen Gesellschaft 23 (1890) 3023-3033. doi:10.1002/cber.189002302232.

[22] K. O. Christe, W. W. Wilson, J. A. Sheehy, J. A. Boatz, N A Novel Homoleptic Polynitrogen Ion as a High Energy Density Material, Angewandte Chemie International Edition 38 (1999) 2004-2009. doi:10.1002/(SICI)1521-3773(19990712)38:13/14¡2004::AIDANIE2004¿3.0.CO;2-7.

[23] S. Fau, R. J. Bartlett, Possible Products of the End-On Addition of $\mathrm{N}_{3}^{-}$ to $\mathrm{N}_{5}^{+}$and Their Stability, The Journal of Physical Chemistry A 105 (2001) 4096-4106. doi:10.1021/jp003970h.

[24] L. Gagliardi, G. Orlandi, S. Evangelisti, B. O. Roos, A theoretical study of the nitrogen clusters formed from the ions $\mathrm{N}_{3}^{-}, \mathrm{N}_{5}^{+}$, and $\mathrm{N}_{5}^{-}$, Journal of Chemical Physics 114 (2001) 10733-10737. doi:10.1063/1.1370063.

[25] S. Evangelisti, T. Leininger, Ionic nitrogen clusters, Journal of Molecular Structure: THEOCHEM 621 (2003) 43-50. doi:10.1016/S01661280(02)00532-8.

[26] D. A. Dixon, D. Feller, K. O. Christe, W. W. Wilson, A. Vij, V. Vij, H. D. B. Jenkins, R. M. Olson, M. S. Gordon, Enthalpies of Formation of Gas-Phase $\mathrm{N}_{3}, \mathrm{~N}_{3}^{-}, \mathrm{N}_{5}^{+}$, and $\mathrm{N}_{5}^{-}$from $\mathrm{Ab}$ Initio Molecular Orbital Theory, Stability Predictions for $\mathrm{N}_{5}^{+} \mathrm{N}_{3}^{-}$and $\mathrm{N}_{5}^{+} \mathrm{N}_{5}^{-}$, and Experimental Evidence for the Instability of $\mathrm{N}_{5}^{+} \mathrm{N}_{3}^{-}$, Journal of the American Chemical Society 126 (2004) 834-843. doi:10.1021/ja0303182.

[27] Y. Li, H. Bai, F. Lin, Y. Huang, Energetics and electronic structures of nitrogen chains encapsulated in zigzag carbon nanotube, Physica E: Low-dimensional Systems and Nanostructures 103 (2018) 444-451. doi:10.1016/j.physe.2018.02.021. 
[28] F. Zheng, C. Wang, P. Zhang, Polymeric Nitrogen Chain Confined Inside a Silicon Carbide Nanotube, Journal of Computational and Theoretical Nanoscience 9 (2012) 1129-1133. doi:10.1166/jctn.2012.2151.

[29] F. Zheng, Y. Yang, P. Zhang, Polymeric Nitrogen Chains Confined in Carbon Nanotube Bundle, International Journal of Modern Physics B 26 (2012) 1250047. doi:10.1142/S0217979212500476.

[30] S. Liu, M. Yao, F. Ma, B. Liu, Z. Yao, R. Liu, T. Cui, B. Liu, High Energetic Polymeric Nitrogen Stabilized in the Confinement of Boron Nitride Nanotube at Ambient Conditions, The Journal of Physical Chemistry C 120 (2016) 16412-16417. doi:10.1021/acs.jpcc.6b04374.

[31] H. Sharma, I. Garg, K. Dharamvir, V. K. Jindal, Structure of Polynitrogen Clusters Encapsulated in $\mathrm{C}_{60}$ : A Density Functional Study, Journal of Physical Chemistry C 114 (2010) 9153-9160. doi:10.1021/jp908755r.

[32] Z. Wu, E. M. Benchafia, Z. Iqbal, X. Wang, $\mathrm{N}_{8}^{-}$Polynitrogen Stabilized on Multi-Wall Carbon Nanotubes for Oxygen-Reduction Reactions at Ambient Conditions, Angewandte Chemie - International Edition 126 (2014) 12763-12767. doi:10.1002/anie.201403060.

[33] E. M. Benchafia, Z. Yao, G. Yuan, T. Chou, H. Piao, X. Wang, Z. Iqbal, Cubic gauche polymeric nitrogen under ambient conditions, Nature Communications 8 (2017) 930. doi:10.1038/s41467-017-01083-5.

[34] P. Gray, T. C. Waddington, Fundamental vibration frequencies and force constants in the azide ion, Transactions of the Faraday Society 53 (1957) 901. doi:10.1039/tf9575300901.

[35] R. Frech, J. C. Decius, Dipolar Coupling and Molecular Vibrations in Crystals. III. Polarizabilities of Molecular Anions and the Internal Field in Some Rhombohedral Crystals, The Journal of Chemical Physics 51 (1969) 5315-5322. doi:10.1063/1.1671951. 
[36] E. Illenberger, P. B. Comita, J. I. Brauman, H.-P. Fenzlaff, M. Heni, N. Heinrich, W. Koch, G. Frenking, Experimental and Theoretical Investigation of the Azide Anion $\left(\mathrm{N}_{3}^{-}\right)$in the Gas Phase, Berichte der Bunsengesellschaft für physikalische Chemie 89 (1985) 10261031. URL: http://doi.wiley.com/10.1002/bbpc.19850891004. doi:10.1002/bbpc.19850891004.

[37] M. Polak, M. Gruebele, R. J. Saykally, Velocity Modulation Laser Spectroscopy of Negative Ions: The $\nu_{3}$ Band of Azide $\left(\mathrm{N}_{3}^{-}\right)$, Journal of the American Chemical Society 109 (1987) 2884-2887. doi:10.1021/ja00244a005.

[38] C. R. Brazier, P. F. Bernath, J. B. Burkholder, C. J. Howard, Fourier transform spectroscopy of the $\nu_{3}$ band of the $\mathrm{N}_{3}$ radical, The Journal of Chemical Physics 89 (1988) 1762-1767. doi:10.1063/1.455122.

[39] R. Tian, J. C. Facelli, J. Michl, Vibrational and electronic spectra of matrix-isolated nitrogen trimer radical and azide, The Journal of Physical Chemistry 92 (1988) 4073-4079. doi:10.1021/j100325a018.

[40] M. Polak, M. Gruebele, G. S. Peng, R. J. Saykally, Velocity modulation infrared laser spectroscopy of negative ions: The (011)-(001) band of azide $\left(\mathrm{N}_{3}^{-}\right)$, The Journal of Chemical Physics 89 (1988) 110-114. doi:10.1063/1.455697.

[41] G. Maroulis, A. Hatzis, A. Haskopoulos, Electric properties of the azide ion calculated with a systematic sequence of Gaussian-type basis sets, Chemical Physics 323 (2006) 451-457. doi:10.1016/j.chemphys.2005.10.020.

[42] P. Sebald, C. Stein, R. Oswald, P. Botschwina, Rovibrational States of $\mathrm{N}_{3}^{-}$and $\mathrm{CO}_{2}$ Up to High $J$ : A Theoretical Study Beyond fc$\operatorname{CCSD}(\mathrm{T})$, The Journal of Physical Chemistry A 117 (2013) 1380613814. doi:10.1021/jp4081806. 
[43] M. Li, J. Owrutsky, M. Sarisky, J. P. Culver, A. Yodh, R. M. Hochstrasser, Vibrational and rotational relaxation times of solvated molecular ions, The Journal of Chemical Physics 98 (1993) 5499-5507. doi:10.1063/1.464899.

[44] M. Ferrario, M. L. Klein, I. R. McDonald, Dynamical behavior of the azide ion in protic solvents, Chemical Physics Letters 213 (1993) 537540. doi:10.1016/0009-2614(93)89156-C.

[45] A. Morita, S. Kato, Vibrational relaxation of azide ion in water: The role of intramolecular charge fluctuation and solvent-induced vibrational coupling, The Journal of Chemical Physics 109 (1998) 5511-5523. doi:10.1063/1.477170.

[46] D. A. Yarne, M. E. Tuckerman, M. L. Klein, Structural and dynamical behavior of an azide anion in water from ab initio molecular dynamics calculations, Chemical Physics 258 (2000) 163-169. doi:10.1016/S03010104(00)00114-2.

[47] Q. Zhong, A. P. Baronavski, J. C. Owrutsky, Vibrational energy relaxation of aqueous azide ion confined in reverse micelles, The Journal of Chemical Physics 118 (2003) 7074-7080. doi:10.1063/1.1562608.

[48] X. Yang, B. Kiran, X.-B. Wang, L.-S. Wang, M. Mucha, P. Jungwirth, Solvation of the Azide Anion $\left(\mathrm{N}_{3}^{-}\right)$in Water Clusters and Aqueous Interfaces: A Combined Investigation by Photoelectron Spectroscopy, Density Functional Calculations, and Molecular Dynamics Simulations, The Journal of Physical Chemistry A 108 (2004) 78207826. doi:10.1021/jp0496396.

[49] L. Bondesson, L. Frediani, H. Ågren, B. Mennucci, Solvation of $\mathrm{N}_{3}^{-}$at the Water Surface: The Polarizable Continuum Model Approach, The Journal of Physical Chemistry B 110 (2006) 11361-11368. doi:10.1021/jp060794p. 
[50] S. Li, J. R. Schmidt, A. Piryatinski, C. P. Lawrence, J. L. Skinner, Vibrational Spectral Diffusion of Azide in Water, The Journal of Physical Chemistry B 110 (2006) 18933-18938. doi:10.1021/jp057568k.

[51] S. Li, J. R. Schmidt, J. L. Skinner, Vibrational energy relaxation of azide in water, Journal of Chemical Physics 125 (2006). doi:10.1063/1.2408421.

[52] S. Li, J. R. Schmidt, S. A. Corcelli, C. P. Lawrence, J. L. Skinner, Approaches for the calculation of vibrational frequencies in liquids: Comparison to benchmarks for azide/water clusters, The Journal of Chemical Physics 124 (2006) 204110. doi:10.1063/1.2200690.

[53] C. H. Kuo, D. Y. Vorobyev, J. Chen, R. M. Hochstrasser, Correlation of the vibrations of the aqueous azide ion with the $\mathrm{O}-\mathrm{H}$ modes of bound water molecules, Journal of Physical Chemistry B 111 (2007) 1402814033. doi:10.1021/jp076503.

[54] G. M. Sando, K. Dahl, J. C. Owrutsky, Vibrational Spectroscopy and Dynamics of Azide Ion in Ionic Liquid and Dimethyl Sulfoxide Water Mixtures, The Journal of Physical Chemistry B 111 (2007) 4901-4909. doi:10.1021/jp067143d.

[55] M. Olschewski, S. Knop, J. Lindner, P. Vöhringer, Vibrational relaxation of azide ions in liquid-to-supercritical water, The Journal of Chemical Physics 134 (2011) 214504. URL: $\quad$ http://aip.scitation.org/doi/10.1063/1.3598108. doi:10.1063/1.3598108.

[56] S. Battaglia, S. Evangelisti, T. Leininger, Confinement of the Pentanitrogen Cation Inside Carbon Nanotubes, in: O. Gervasi, B. Murgante, S. Misra, E. Stankova, C. M. Torre, A. M. A. Rocha, D. Taniar, B. O. Apduhan, E. Tarantino, Y. Ryu (Eds.), Computational Science and Its 
Applications - ICCSA 2018, volume 10964 of Lecture Notes in Computer Science, Springer International Publishing, Cham, 2018, pp. 579-592. doi:10.1007/978-3-319-95174-4.

[57] I. T. Todorov, W. Smith, K. Trachenko, M. T. Dove, DL POLY 3: New dimensions in molecular dynamics simulations via massive parallelism, Journal of Materials Chemistry (2006). doi:10.1039/b517931a.

[58] I. T. Todorov, W. Smith, DL POLY 4, 2011.

[59] S. Grimme, J. Antony, S. Ehrlich, H. Krieg, A consistent and accurate ab initio parametrization of density functional dispersion correction (DFTD) for the 94 elements H-Pu, Journal of Chemical Physics 132 (2010). doi:10.1063/1.3382344.

[60] S. Grimme, S. Ehrlich, L. Goerigk, Effect of the damping function in dispersion corrected density functional theory, Journal of Computational Chemistry 32 (2011) 1456-1465. doi:10.1002/jcc.21759.

[61] T. H. Dunning Jr., Gaussian basis sets for use in correlated molecular calculations. I. The atoms boron through neon and hydrogen, The Journal of Chemical Physics 90 (1989) 1007-1023. doi:10.1063/1.456153.

[62] R. A. Kendall, T. H. Dunning Jr., R. J. Harrison, Electron affinities of the first-row atoms revisited. Systematic basis sets and wave functions and wave functions, Journal of Chemical Physics 96 (1992) 6796-6806. doi:10.1063/1.462569.

[63] Z. Zhou, M. Steigerwald, M. Hybertsen, L. Brus, R. A. Friesner, Electronic Structure of Tubular Aromatic Molecules Derived from the Metallic $(5,5)$ Armchair Single Wall Carbon Nanotube, Journal of the American Chemical Society 126 (2004) 3597-3607. doi:10.1021/ja039294p. 
[64] A. Galano, On the influence of diameter and length on the properties of armchair single-walled carbon nanotubes: A theoretical chemistry approach, Chemical Physics 327 (2006) 159-170. doi:10.1016/j.chemphys.2006.04.019.

[65] M. J. Frisch, G. W. Trucks, H. B. Schlegel, G. E. Scuseria, M. A. Robb, J. R. Cheeseman, G. Scalmani, V. Barone, B. Mennucci, G. A. Petersson, H. Nakatsuji, M. Caricato, X. Li, H. P. Hratchian, A. F. Izmaylov, J. Bloino, G. Zheng, J. L. Sonnenberg, M. Hada, M. Ehara, K. Toyota, R. Fukuda, J. Hasegawa, M. Ishida, T. Nakajima, Y. Honda, O. Kitao, H. Nakai, T. Vreven, J. A. Montgomery Jr., J. E. Peralta, F. Ogliaro, M. Bearpark, J. J. Heyd, E. Brothers, K. N. Kudin, V. N. Staroverov, R. Kobayashi, J. Normand, K. Raghavachari, A. Rendell, J. C. Burant, S. S. Iyengar, J. Tomasi, M. Cossi, N. Rega, J. M. Millam, M. Klene, J. E. Knox, J. B. Cross, V. Bakken, C. Adamo, J. Jaramillo, R. Gomperts, R. E. Stratmann, O. Yazyev, A. J. Austin, R. Cammi, C. Pomelli, J. W. Ochterski, R. L. Martin, K. Morokuma, V. G. Zakrzewski, G. A. Voth, P. Salvador, J. J. Dannenberg, S. Dapprich, A. D. Daniels, Ö. Farkas, J. B. Foresman, J. V. Ortiz, J. Cioslowski, D. J. Fox, Gaussian 09 Revision D.01, ???? URL: http://www.gaussian.com.

[66] S. F. Boys, F. Bernardi, The calculation of small molecular interactions by the differences of separate total energies. Some procedures with reduced errors, Molecular Physics 19 (1970) 553-566. doi:10.1080/00268977000101561.

[67] F. Weigend, Accurate Coulomb-fitting basis sets for $\mathrm{H}$ to Rn, Physical Chemistry Chemical Physics 8 (2006) 1057. doi:10.1039/b515623h.

[68] F. Neese, Software update: the ORCA program system, version 4.0, Wiley Interdisciplinary Reviews: Computational Molecular Science (2017) e1327. doi:10.1002/wcms.1327. 
[69] S. Grimme, Improved second-order Møller-Plesset perturbation theory by separate scaling of parallel- and antiparallel-spin pair correlation energies, The Journal of Chemical Physics 118 (2003) 9095-9102. doi:10.1063/1.1569242.

[70] C. Riplinger, F. Neese, An efficient and near linear scaling pair natural orbital based local coupled cluster method, The Journal of Chemical Physics 138 (2013) 034106. doi:10.1063/1.4773581.

[71] C. Riplinger, P. Pinski, U. Becker, E. F. Valeev, F. Neese, Sparse maps-A systematic infrastructure for reduced-scaling electronic structure methods. II. Linear scaling domain based pair natural orbital coupled cluster theory, The Journal of Chemical Physics 144 (2016) 024109. doi:10.1063/1.4939030.

[72] A. Halkier, T. Helgaker, P. Jørgensen, W. Klopper, H. Koch, J. Olsen, A. K. Wilson, Basis-set convergence in correlated calculations on Ne, N2, and H2O, Chemical Physics Letters 286 (1998) 243252. URL: http://www. sciencedirect.com/science/article/pii/ S0009261498001110. doi:10.1016/S0009-2614(98)00111-0.

[73] S. Grimme, C. Mück-Lichtenfeld, J. Antony, Noncovalent Interactions between Graphene Sheets and in Multishell (Hyper)Fullerenes, The Journal of Physical Chemistry C 111 (2007) 11199-11207. doi:10.1021/jp0720791.

[74] F. Pirani, S. Brizi, L. F. Roncaratti, P. Casavecchia, D. Cappelletti, F. Vecchiocattivi, Beyond the Lennard-Jones model: a simple and accurate potential function probed by high resolution scattering data useful for molecular dynamics simulations., Physical Chemistry Chemical Physics 10 (2008) 5489-5503. doi:10.1039/b808524b.

[75] N. Faginas Lago, F. Huarte Larrañaga, M. Albertí, On the suitability of the ILJ function to match different formulations of the electrostatic 
potential for water-water interactions, The European Physical Journal D 55 (2009) 75-85. doi:10.1140/epjd/e2009-00215-5.

[76] M. Bartolomei, E. Carmona-Novillo, M. I. Hernández, J. CamposMartínez, F. Pirani, G. Giorgi, K. Yamashita, Penetration Barrier of Water through Graphynes' Pores: First-Principles Predictions and Force Field Optimization, The Journal of Physical Chemistry Letters 5 (2014) 751-755. doi:10.1021/jz4026563.

[77] M. Albertí, A. Aguilar, J. M. Lucas, F. Pirani, C. Coletti, N. Re, AtomBond Pairwise Additive Representation for Halide-Benzene Potential Energy Surfaces: an Ab Initio Validation Study, The Journal of Physical Chemistry A 113 (2009) 14606-14614. doi:10.1021/jp904329r.

[78] M. Albertí, A. Aguilar, J. M. Lucas, F. Pirani, A Generalized Formulation of Ion- $\pi$ Electron Interactions: Role of the Nonelectrostatic Component and Probe of the Potential Parameter Transferability, The Journal of Physical Chemistry A 114 (2010) 11964-11970. doi:10.1021/jp105763h.

[79] M. Bartolomei, E. Carmona-Novillo, M. I. Hernández, J. Campos-Martínez, F. Pirani, G. Giorgi, Graphdiyne Pores: "Ad Hoc" Openings for Helium Separation Applications, The Journal of Physical Chemistry C 118 (2014) 2996629972. URL: http://pubs.acs.org/doi/10.1021/jp510124e. doi:10.1021/jp510124e.

[80] N. Faginas-Lago, D. Yeni, F. Huarte, Y. Wang, M. Alcamí, F. Martin, Adsorption of Hydrogen Molecules on Carbon Nanotubes Using Quantum Chemistry and Molecular Dynamics, The Journal of Physical Chemistry A 120 (2016) 6451-6458. doi:10.1021/acs.jpca.5b12574.

[81] A. W. Hauser, M. P. de Lara-Castells, Carbon Nanotubes Immersed in Superfluid Helium: The Impact of Quantum Confinement on Wetting 
and Capillary Action, The Journal of Physical Chemistry Letters 7 (2016) 4929-4935. doi:10.1021/acs.jpclett.6b02414.

[82] A. W. Hauser, A. O. Mitrushchenkov, M. P. De Lara-Castells, Quantum Nuclear Motion of Helium and Molecular Nitrogen Clusters in Carbon Nanotubes, Journal of Physical Chemistry C 121 (2017) 3807-3821. doi:10.1021/acs.jpcc.6b12959.

[83] J. Wilson, N. Faginas-Lago, J. Vekeman, I. G. Cuesta, J. Sánchez-Marín, A. Sánchez de Merás, Modeling the Interaction of Carbon Monoxide with Flexible Graphene: From Coupled Cluster Calculations to Molecular-Dynamics Simulations, ChemPhysChem 19 (2018) 774-783. doi:10.1002/cphc.201701387.

[84] J. Vekeman, I. G. Cuesta, N. Faginas-Lago, J. Wilson, J. SánchezMarín, A. Sánchez de Merás, Potential models for the simulation of methane adsorption on graphene: development and $\operatorname{CCSD}(\mathrm{T})$ benchmarks, Physical Chemistry Chemical Physics 20 (2018) 25518-25530. doi:10.1039/C8CP03652G.

[85] A. Stone, The Theory of Intermolecular Forces, Oxford University Press, 2013. doi:10.1093/acprof:oso/9780199672394.001.0001.

[86] R. Cambi, D. Cappelletti, G. Liuti, F. Pirani, Generalized correlations in terms of polarizability for van der Waals interaction potential parameter calculations, The Journal of Chemical Physics 95 (1991) 1852-1861. doi:10.1063/1.461035.

[87] D. Cappelletti, G. Liuti, F. Pirani, Generalization to ion-neutral systems of the polarizability correlations for interaction potential parameters, Chemical Physics Letters 183 (1991) 297-303. doi:10.1016/00092614(91)80066-7. 
[88] V. Aquilanti, D. Cappelletti, F. Pirani, Range and strength of interatomic forces: dispersion and induction contributions to the bonds of dications and of ionic molecules, Chemical Physics 209 (1996) 299-311. doi:10.1016/0301-0104(96)00163-2.

[89] C. Le Borgne, B. Illien, M. Beignon, M. Chabanel, Ion association of alkali and alkaline earth metal azides in dimethylsulfoxide. Infrared spectrometry and ab initio calculations, Physical Chemistry Chemical Physics 1 (1999) 4701-4706. doi:10.1039/a905936i.

[90] A. Gavezzotti, Calculation of Intermolecular Interaction Energies by Direct Numerical Integration over Electron Densities. 2. An Improved Polarization Model and the Evaluation of Dispersion and Repulsion Energies, The Journal of Physical Chemistry B 107 (2003) 2344-2353. doi:10.1021/jp022288f.

[91] F. Torrens, Effect of type, size and deformation on the polarizability of carbon nanotubes from atomic increments, Nanotechnology 15 (2004) S259-S264. doi:10.1088/0957-4484/15/4/027.

[92] A. E. Reed, R. B. Weinstock, F. Weinhold, Natural population analysis, The Journal of Chemical Physics 83 (1985) 735-746. doi:10.1063/1.449486.

[93] T. Tamayo-Mendoza, C. Kreisbeck, R. Lindh, A. Aspuru-Guzik, Automatic Differentiation in Quantum Chemistry with Applications to Fully Variational Hartree-Fock, ACS Central Science 4 (2018) 559-566. doi:10.1021/acscentsci.7b00586. 
Table 1: Correlation energy contributions to the interaction energy of $\mathrm{N}_{3}^{-} @ \mathrm{CNT}(5,5)$ for different basis set sizes. All values are in $\mathrm{kcal} / \mathrm{mol}$.

\begin{tabular}{ccccc}
\hline Basis & cc-pVDZ & cc-pVTZ & cc-pVQZ & CBS \\
\hline$E_{\text {int }}$ & -30.93 & -33.47 & -34.14 & -34.62 \\
\hline
\end{tabular}


Table 2: Interaction energy fitting parameters.

\begin{tabular}{lcc}
\hline Method & $a(\Lambda \rightarrow \infty)$ & $b$ \\
\hline RI-SCS-MP2/cc-pVDZ & -64.75 & 4.11 \\
DLPNO-CCSD(T)/cc-pVTZ & -65.93 & 2.43 \\
\hline
\end{tabular}


Table 3: Potential parameters for carbon and carbon-hydrogen interactions. Polarizability $(\bar{\alpha})$ given in $\AA^{3}$, energies $(\epsilon)$ in $\mathrm{meV}$ and distances $\left(r_{m}\right)$ in $\AA$.

\begin{tabular}{cccccc}
\hline & & \multicolumn{2}{c}{$\mathrm{C}-\mathrm{N}_{\text {ext }}$} & \multicolumn{2}{c}{ C- $\mathrm{N}_{\text {int }}$} \\
\cline { 3 - 6 }$(m, m)$ & $\bar{\alpha}$ & $\epsilon$ & $r_{m}$ & $\epsilon$ & $r_{m}$ \\
\hline$(5,5)$ & 1.136 & 5.074 & 3.984 & 3.469 & 3.805 \\
$(7,7)$ & 1.179 & 5.163 & 3.991 & 3.514 & 3.814 \\
$(9,9)$ & 1.219 & 5.243 & 3.997 & 3.554 & 3.822 \\
\hline
\end{tabular}


Table 4: Potential parameters for hydrogen and hydrogen-nitrogen interactions. Polarizability $(\bar{\alpha})$ given in $\AA^{3}$, energies $(\epsilon)$ in meV and distances $\left(r_{m}\right)$ in $\AA$.

\begin{tabular}{cccccc}
\hline & & \multicolumn{2}{c}{$\mathrm{H}-\mathrm{N}_{\text {ext }}$} & \multicolumn{2}{c}{$\mathrm{H}-\mathrm{N}_{\text {int }}$} \\
\cline { 3 - 6 } & $\bar{\alpha}$ & $\epsilon$ & $r_{m}$ & $\epsilon$ & $r_{m}$ \\
\hline$(m, m)$ & 0.380 & 2.827 & 3.644 & 2.431 & 3.348 \\
\hline
\end{tabular}




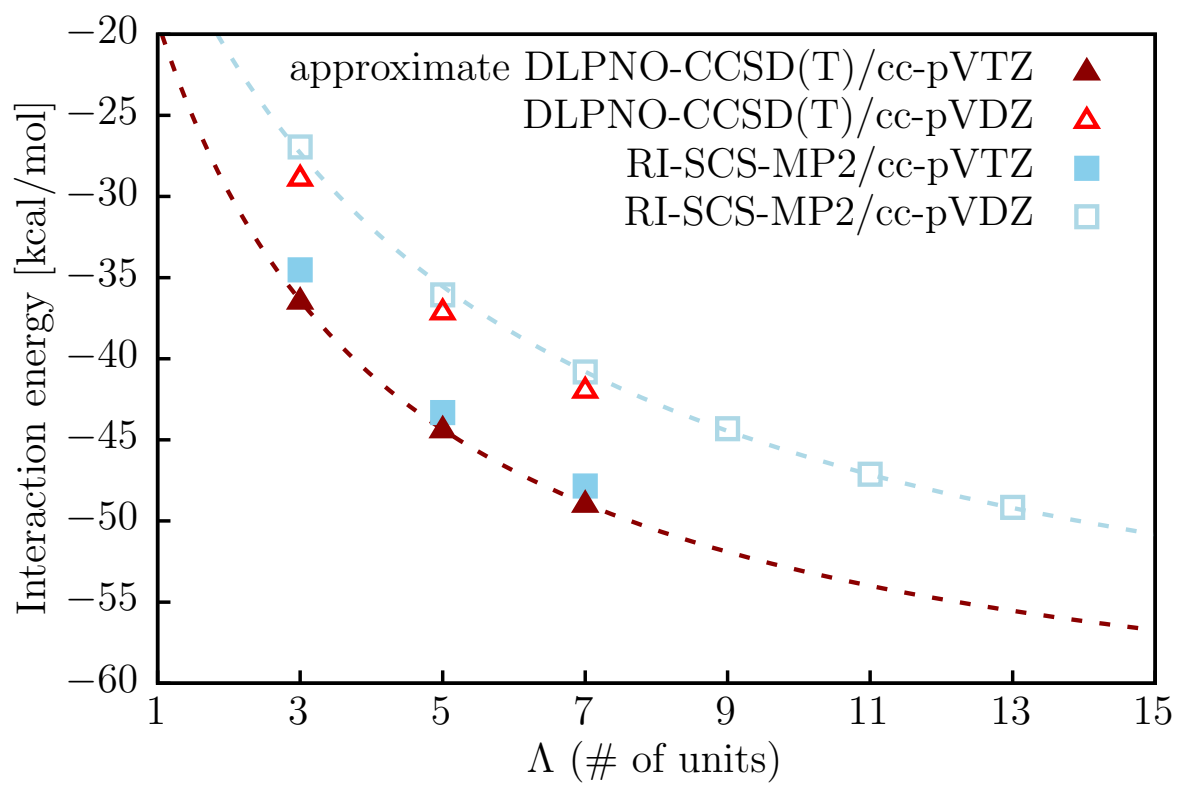

Figure 1: Interaction energy as a function of the number of units $\Lambda$. 


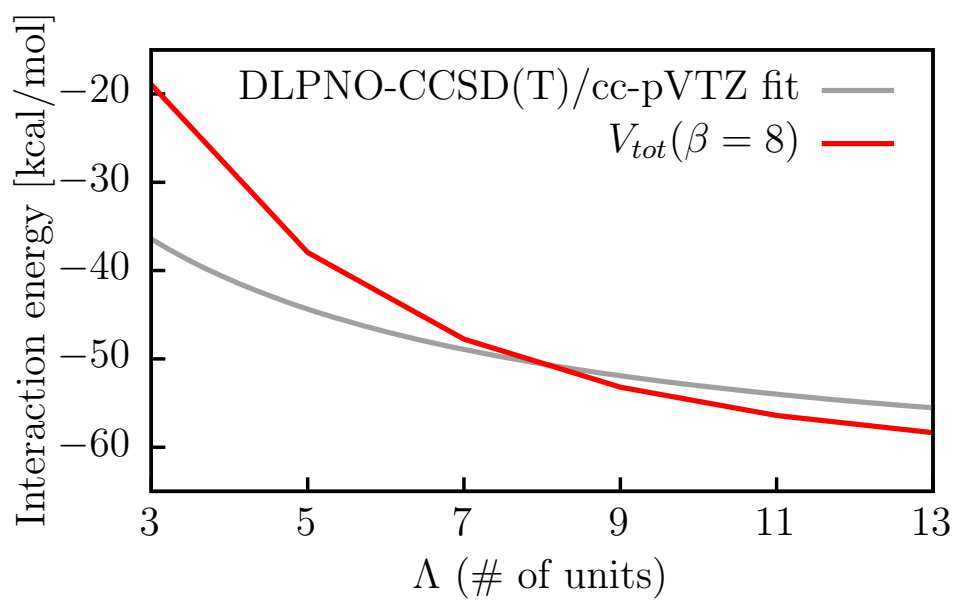

Figure 2: Comparison of classical and ab initio interaction energies as a function of the number of units $\Lambda$. 


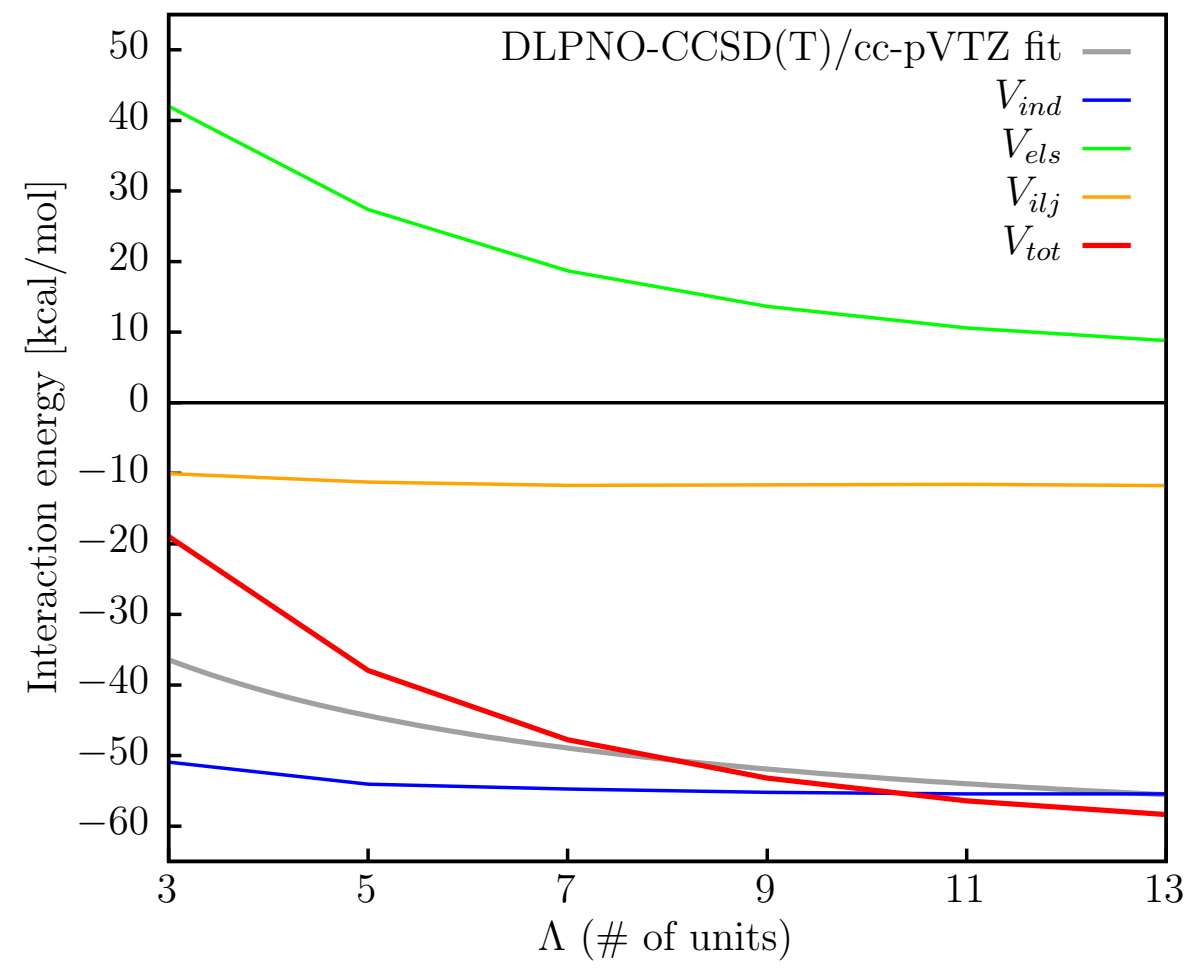

Figure 3: Individual contributions and total potential energy along with the best $a b$ initio fit to the interaction energy as a function of the number of units $\Lambda$. 


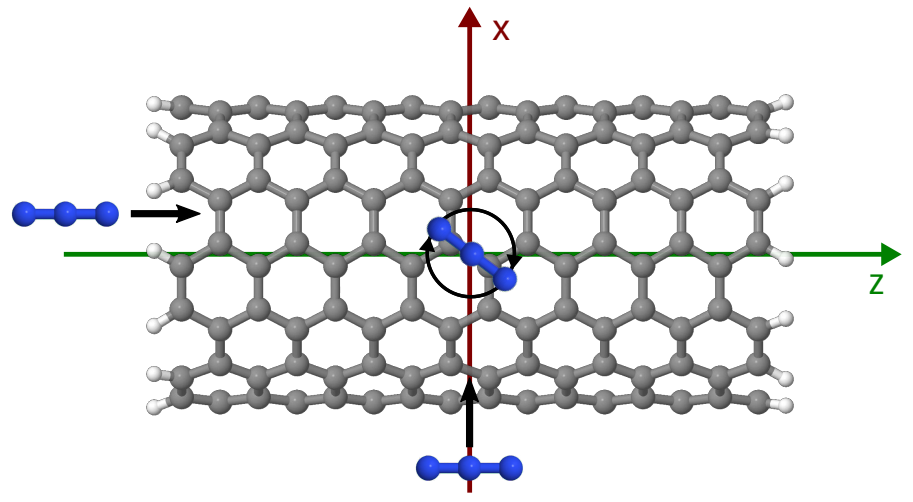

Figure 4: Scheme showing the three different ways in which the azide anion is moved to generate the energy profiles. 


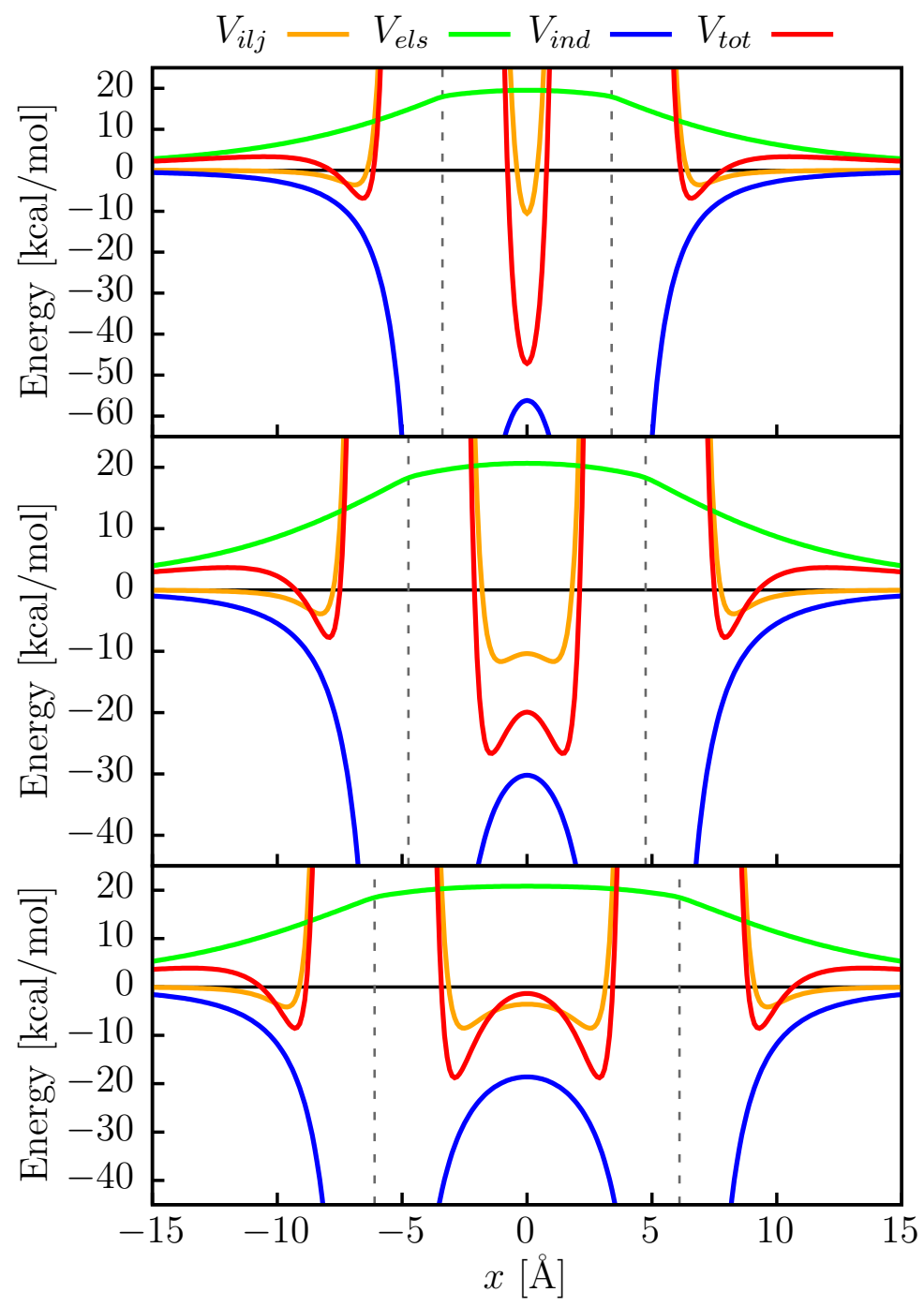

Figure 5: Potential energy profile of the scan of an azide anion approaching a carbon nanotube wall. From top to bottom, the profiles refer to $(5,5),(7,7)$ and $(9,9)$ CNTs, respectively. The dashed vertical lines show the position of the nanotube wall, thus, in each plot, the distance between them represents the CNT diameter, which increases from top to bottom according to the nanotube chiral indices. 


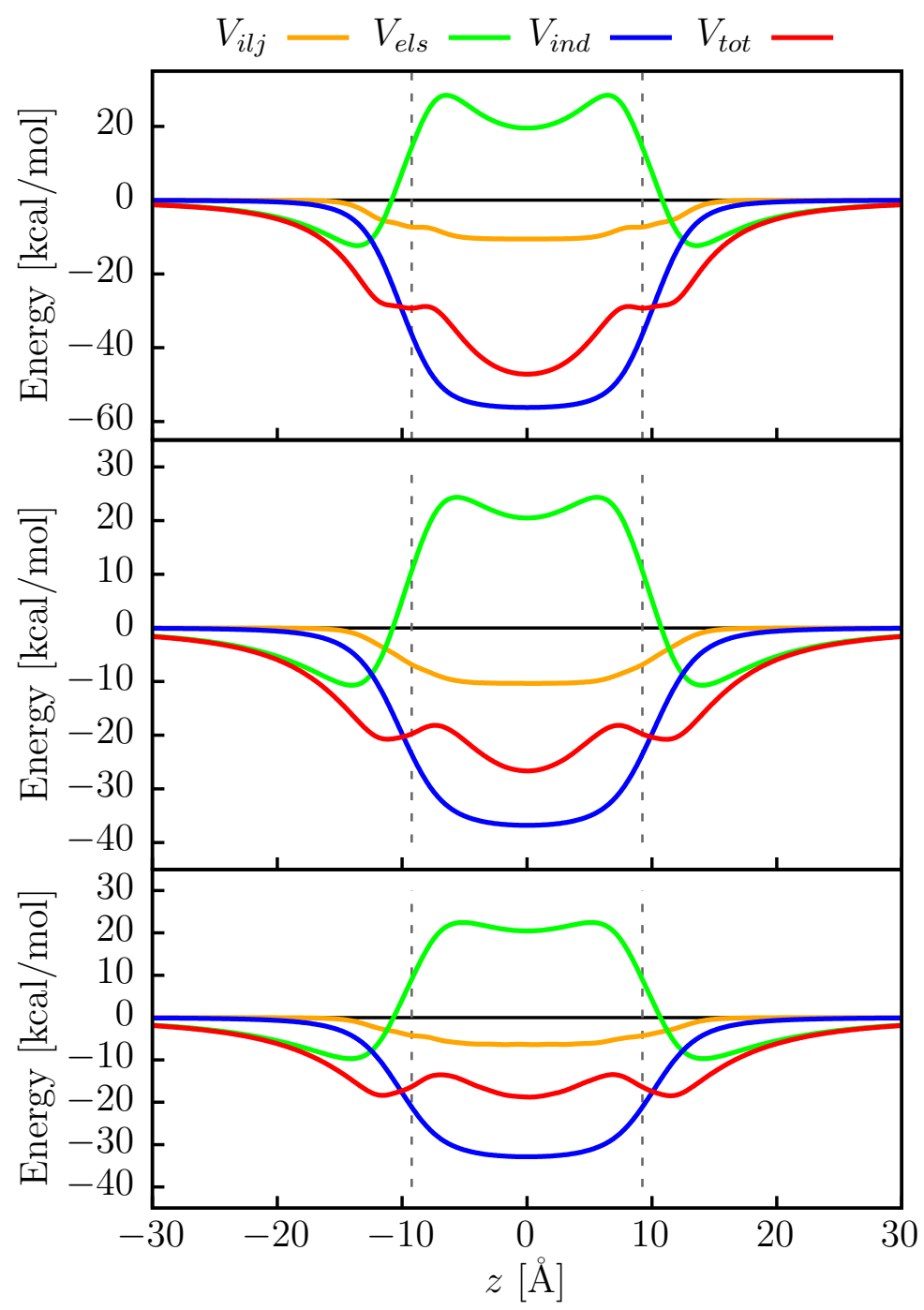

Figure 6: Potential energy profile of the scan of an azide anion approaching a carbon nanotube along its principal axis. From top to bottom, the profiles refer to $(5,5),(7,7)$ and $(9,9)$ CNTs, respectively. The horizontal axis is aligned with the principal axis of the nanotubes and the dashed vertical lines show the position of the nanotube openings. Therefore, in each plot, the distance between them represents the CNT length, which remains the same from top to bottom as the nanotube length is the same for three cases shown here. 


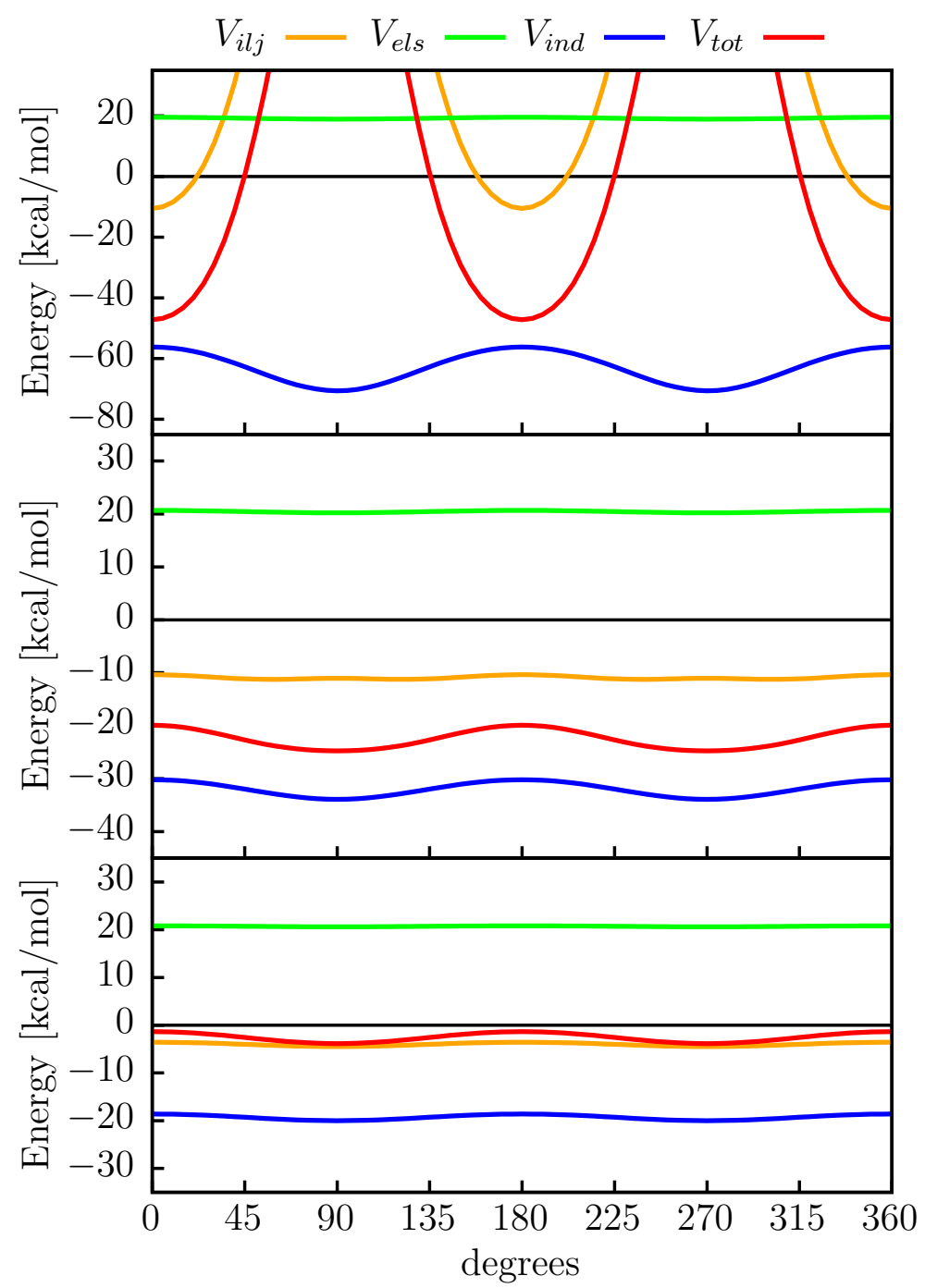

Figure 7: Potential energy profile of the scan of an azide anion rotating inside a carbon nanotube. From top to bottom, the profiles refer to $(5,5),(7,7)$ and $(9,9)$ CNTs. The horizontal axis represents the angle of rotation of the azide anion around its central nitrogen atom. 


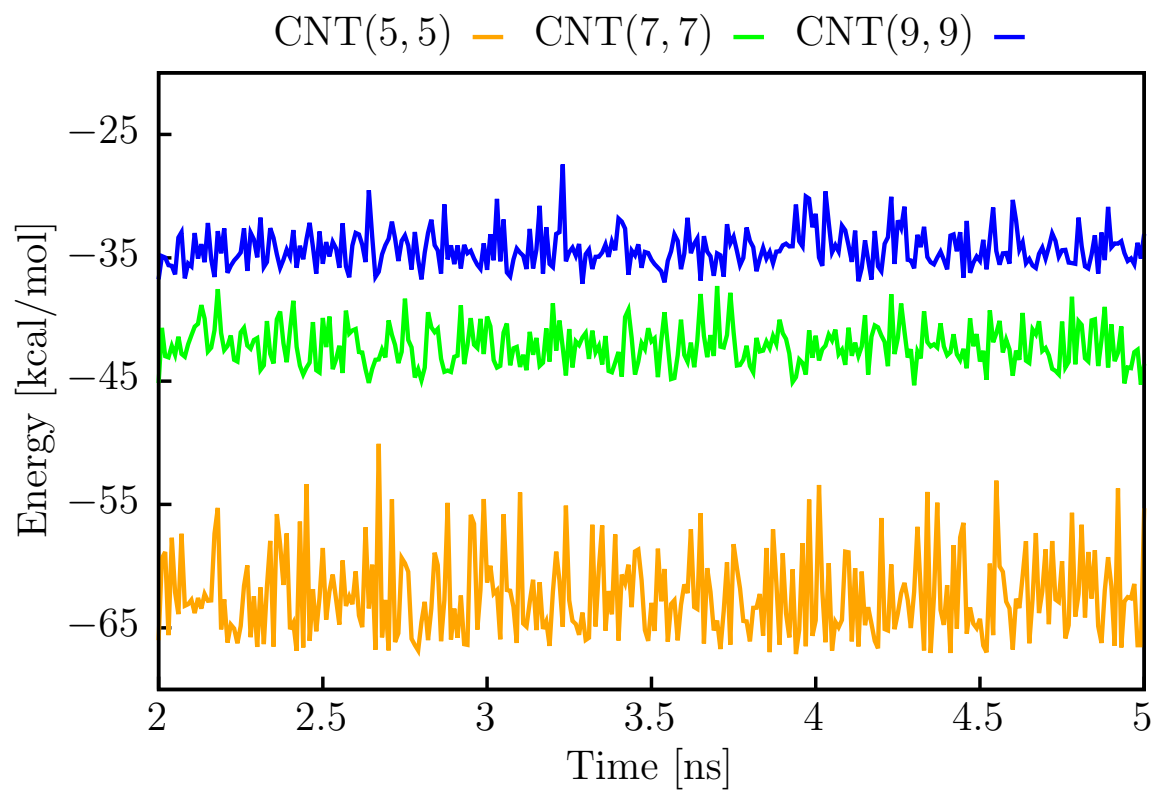

Figure 8: Average adsorption energy of the azide anion confined inside carbon nanotubes of different diameters as a function of the simulation time. 


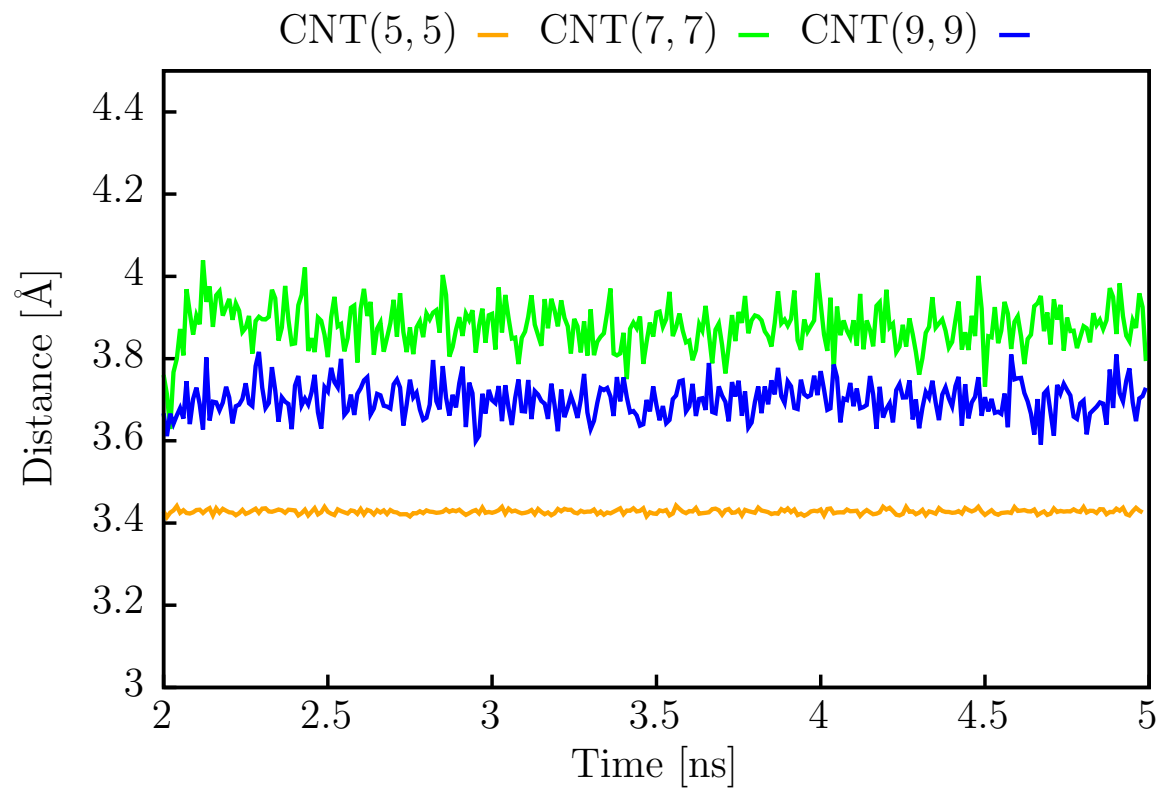

Figure 9: Average minimum distance between the azide anion and the carbon nanotube wall as a function of the simulation time for nanotubes of different diameters. 